\title{
Jackson Integrals of Jordan-Pochhammer Type and Quantum Knizhnik-Zamolodchikov Equations
}

\author{
Atsushi Matsuo \\ Department of Mathematics, Nagoya University, Nagoya 464, Japan
}

Received February 1, 1992

\begin{abstract}
We show that the $q$-difference systems satisfied by Jackson integrals of Jordan-Pochhammer type give a class of the quantum Knizhnik-Zamolodchikov equation for $U_{q}\left(\widehat{\mathfrak{I l}}_{2}\right)$ in the sense of Frenkel and Reshetikhin.
\end{abstract}

\section{Introduction}

One of the most interesting features of the Knizhnik-Zamolodchikov equation originated in conformal field theory is the relation between its connection matrix and the trigonometric solutions of the quantum Yang-Baxter equation [TK, K, D]. It is related to the fact that certain hypergeometric type integrals give solutions to the Knizhnik-Zamolodchikov equation [DJMM, Ma, Ch, SV], etc. This fact is also looked at from the viewpoint of the free field realization, e.g. [Ku, ATY]. Besides them, the structure of the hypergeometric type integrals had been studied, e.g. [A1, A2]. Recently it attracts attention to construct a $q$-analogue of these theories.

The Jackson integrals of Jordan-Pochhammer type are the simplest multivariable generalizations of Heine's basic hypergeometric function which is a $q$-analogue of Gauss' hypergeometric function. They satisfy a system of first order $q$-difference equations, whose connection problem was solved by Mimachi [Mi]. Recently Aomoto and others [AKM] showed that the connection matrix determined by Mimachi is related to the ABF-solution of the quantum Yang-Baxter equation $[\mathrm{ABF}]$. On the other hand, Frenkel and Reshetikhin [FR] studied a $q$-analogue of the chiral vertex operators of the WZNW model, along the line of Tsuchiya and Kanie [TK]. In particular, they introduced a $q$-difference system called the quantum Knizhnik-Zamolodchikov equation, and discussed the relation of the connection matrix with elliptic solutions of the quantum Yang-Baxter equation. Then it seems possible to understand the result of $[\mathrm{AKM}]$ in the framework of Frenkel and Reshetikhin.

In this article, we shall explicitly give solutions to a certain class of the quantum Knizhnik-Zamolodchikov equation for $U_{q}\left(\widehat{\mathfrak{s}}_{2}\right)$ by Jackson integrals of JordanPochhammer type. More precisely, we show that the $q$-difference system for the 
Jackson integrals of Jordan-Pochhammer type is written in terms of trigonometric quantum $R$-matrix, and that this equation gives a class of the quantum Knizhnik-Zamolodchikov equation. When $q$ goes to 1 , our expressions of solutions go to the integral solutions of the Knizhnik-Zamolodchikov equation given by $[\mathrm{Ch}]$ in the trigonometric form.

The paper is organized as follows. In Sect. 2, we write the $q$-difference equation for Jackson integrals of Jordan-Pochhammer type, whose proof will be given in Sect. 4. In Sect. 3, we identify the equation with the quantum KnizhnikZamolodchikov equation. In Sect. 5, we give some comments on the connection problem according to current literatures.

\section{2. $q$-Difference System for Jackson Integrals}

Let $p$ be a fixed complex number such as $0<|p|<1$. Let us denote

$$
(a)_{\infty}=\prod_{n=0}^{\infty}\left(1-a p^{n}\right)
$$

as usual. For a value $s \in \mathbf{C}^{*}$ and for a function $\phi(t)$, we define

$$
\int_{0}^{s \infty} \phi(t) d_{p} t=s(1-p) \sum_{n=-\infty}^{\infty} \phi\left(s p^{n}\right) p^{n}
$$

whenever it is convergent. This is called the Jackson integral along a $q$-interval $[0, s \infty]$, which is a $q$-analogue of the ordinary integration. The $q$-shift operator $T_{k}$ is defined by

$$
\left(T_{k} F\right)\left(x_{1}, \ldots, x_{n}\right)=F\left(x_{1}, \ldots, p x_{k}, \ldots, x_{n}\right)
$$

for a function $F\left(x_{1}, \ldots, x_{n}\right)$.

Now consider the Jackson integral of Jordan-Pochhammer type:

$$
F_{0}(x)=\int_{0}^{s \infty} t^{\beta-1} \prod_{1 \leqq j \leqq n} \frac{\left(t / x_{j}\right)_{\infty}}{\left(p^{\beta_{j}} t / x_{j}\right)_{\infty}} d_{p} t
$$

where $\beta_{j}$ are complex parameters and $x=\left(x_{1}, \ldots, x_{n}\right)$ is a variable in $\left(\mathbf{C}^{\times}\right)^{n}$. We are interested in the $q$-difference system associated with $F_{0}$. Take the set of functions $\left(F_{1}, \ldots, F_{n}\right)$ defined by

$$
F_{i}(x)=\int_{0}^{s \infty} \Phi_{i}(t) d_{p} t
$$

where, for each $i=0, \ldots, n$, we have set

$$
\Phi_{i}(t)=t^{\beta-1} \frac{\prod_{j=1}^{i}\left(p t / x_{j}\right)_{\infty} \prod_{j=i+1}^{n}\left(t / x_{j}\right)_{\infty}}{\prod_{j=1}^{i-1}\left(p^{\beta_{j}+1} t / x_{j}\right)_{\infty} \prod_{j=i}^{n}\left(p^{\beta_{j}} t / x_{j}\right)_{\infty}} .
$$

Let us calculate the $q$-difference system satisfied by $F_{i}$. We set

$$
x_{i j}=\left\{\begin{array}{cl}
x_{i} / x_{j} & \text { if } i<j \\
1 & \text { if } i=j \\
p x_{i} / x_{j} & \text { if } i>j
\end{array}\right.
$$

Then the result is summarized as the following proposition. 
Proposition 1. We define the $n \times n$ matrix $A_{k}$ with entries $a_{i j}^{k}$ as follows. If $i=j \neq k$ then

$$
a_{i j}^{k}=\frac{x_{k i}-1}{x_{k i}-p^{\beta_{k}}}
$$

If $i<j \leqq k$ or $k \leqq i<j$ then

$$
a_{i j}^{k}=\frac{\left(1-p^{\beta_{i}}\right) x_{k i}}{x_{k i}-p^{\beta_{k}}} \frac{1-p^{\beta_{k}}}{x_{k j}-p^{\beta_{k}}} \prod_{l=i+1}^{j-1} \frac{p^{\beta_{l}} x_{k l}-p^{\beta_{k}}}{x_{k l}-p^{\beta_{k}}} .
$$

If $j \leqq k \leqq i$ then

$$
a_{i j}^{k}=p^{\beta} \frac{1-p^{\beta_{k}}}{x_{k j}-p^{\beta_{k}}} \frac{\left(1-p^{\beta_{i}}\right) x_{k i}^{j-1}}{x_{k i}-p^{\beta_{k}}} \prod_{l=1}^{j} \frac{p^{\beta_{l}} x_{k l}-p^{\beta_{k}}}{x_{k l}-p^{\beta_{k}}} \prod_{l=i+1}^{n} \frac{\mathrm{p}^{\beta_{l}} x_{k l}-p^{\beta_{k}}}{x_{k l}-p^{\beta_{k}}} .
$$

Otherwise $a_{i j}^{k}=0$.

Then we have

$$
\left(T_{k} F_{1}, \ldots, T_{k} F_{n}\right)=\left(F_{1}, \ldots, F_{n}\right) A_{k} .
$$

Remark. For each $i, j(i \neq j)$, let $S_{i, j}$ denote the $n \times n$-matrix defined by

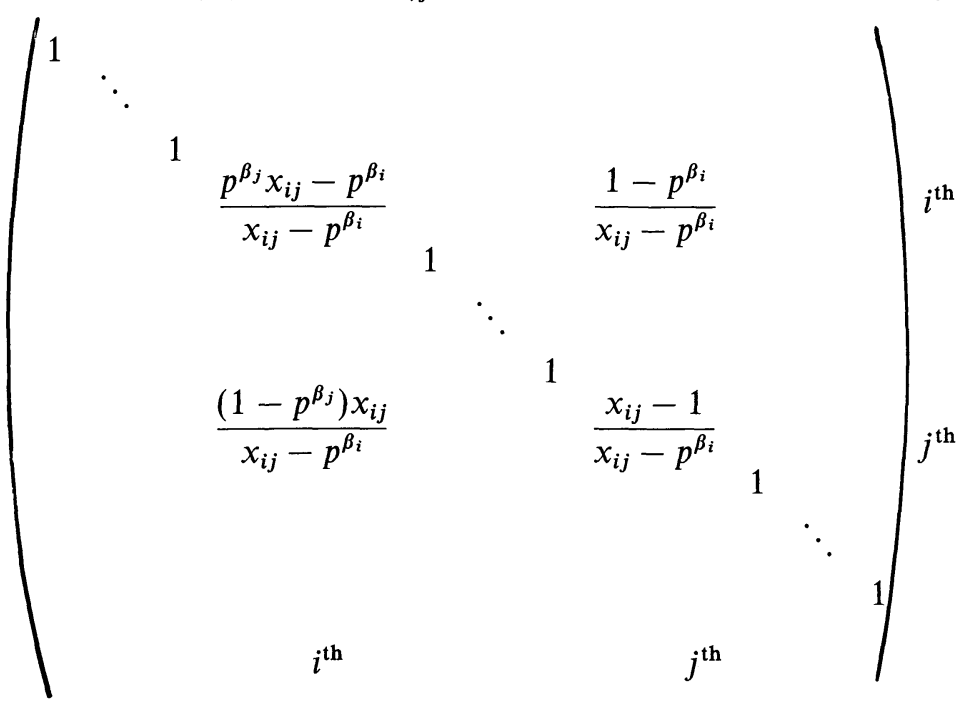

We also consider the $n \times n$-matrix $P_{k}$ defined by

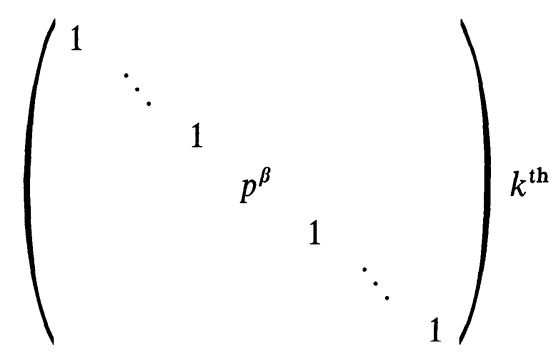


Then, by an explicit calculation, we see

$$
A_{i}=S_{k, k+1} \ldots S_{k, n} P_{k} S_{k, 1} \ldots S_{k, k-1} \text {. }
$$

Matrices $S_{i, j}$ form a set of unitary quantum $R$-matrices. Namely we have

$$
S_{i, j}\left(T_{i} S_{j, i}\right)=\mathrm{id}, \quad \text { and } S_{1,2} S_{2,3} S_{1,3}=S_{1,3} S_{2,3} S_{1,2} \text {. }
$$

Finally, let us discuss the relation among $F_{0}, \ldots, F_{n}$.

Proposition 2. We put $\beta_{0}=-\beta-\left(\beta_{1}+\cdots+\beta_{n}\right)$. Then the following relation holds:

$$
\sum_{i=0}^{n} p^{\beta_{i+1}+\cdots+\beta_{n}}\left(1-p^{\beta_{i}}\right) F_{i}=0 .
$$

Therefore $F_{0}$ is recovered from $F_{1}, \ldots, F_{n}$ if $p^{\beta_{0}} \neq 1$.

Remark. The identity (2.17) is a q-analogue of Aomoto's linear relation in the sense of [A2] and [DJMM].

\section{Comparison with the Quantum Knizhnik-Zamolodchikov Equations}

Let us briefly review the quantum enveloping algebra and the trigonometric $R$-matrix in the case of $\widehat{\mathfrak{s l}}_{2}$. The quantum enveloping algebra $\hat{U}_{q}=U_{q}\left(\widehat{\mathfrak{s l}}_{2}\right)$ is defined as an algebra with the generators:

$$
X_{0}^{ \pm}, X_{1}^{ \pm}, K_{0}^{ \pm 1}, K_{1}^{ \pm 1}
$$

and the relations:

$$
\begin{gathered}
K_{0} K_{1}=K_{1} K_{0}, \quad K_{0} K_{0}^{-1}=K_{1} K_{1}^{-1}=1, \\
K_{i} X_{i}^{ \pm} K_{i}^{-1}=q^{ \pm 2} X_{i}^{ \pm}, \quad K_{i} X_{j}^{ \pm} K_{i}^{-1}=q^{\mp 2} X_{j}^{ \pm}(i \neq j), \\
{\left[X_{i}^{+}, X_{j}^{-}\right]=\delta_{i j} \frac{K_{i}-K_{i}^{-1}}{q-q^{-1}},} \\
\left(X_{i}^{ \pm}\right)^{3} X_{j}^{ \pm}-\left(q^{2}+1+q^{-2}\right)\left(X_{i}^{ \pm}\right)^{2} X_{j}^{ \pm} X_{i}^{ \pm}+\left(q^{2}+1+q^{-2}\right) X_{i}^{ \pm} X_{j}^{ \pm}\left(X_{i}^{ \pm}\right)^{2} \\
-X_{j}^{ \pm}\left(X_{i}^{ \pm}\right)^{3}=0 \quad(i \neq j) .
\end{gathered}
$$

Here, $q$ denotes a general complex parameter. The comultiplication $\Delta: \hat{U}_{q} \rightarrow \hat{U}_{q} \otimes \hat{U}_{q}$ is defined by

$$
\begin{gathered}
\Delta\left(X_{i}^{+}\right)=X_{i}^{+} \otimes 1+K_{i}^{-1} \otimes X_{i}^{+}, \\
\Delta\left(X_{i}^{-}\right)=X_{i}^{-} \otimes K_{i}+1 \otimes X_{i}^{-}, \quad \Delta\left(K_{i}\right)=K_{i} \otimes K_{i} .
\end{gathered}
$$

We put $\Delta^{\prime}=\sigma \circ \Delta$ where $\sigma(a \otimes b)=b \otimes a$ in $\hat{U}_{q} \otimes \hat{U}_{q}$. Next we consider the subalgebra $U_{q}=U_{q}\left(\mathfrak{s l}_{2}\right)$ generated by $X^{ \pm}=X_{1}^{ \pm}, K^{ \pm}=K_{1}^{ \pm}$. For each $x \in \mathbf{C}^{*}$, we define the algebra homomorphism $\varphi_{x}: \hat{U}_{q} \rightarrow U_{q}$ by

$$
\begin{array}{cl}
\varphi_{x}\left(X_{0}^{ \pm}\right)=x^{ \pm 1} X^{\mp}, & \varphi_{x}\left(X_{1}^{ \pm}\right)=X^{ \pm}, \\
\varphi_{x}\left(K_{0}\right)=K^{-1}, & \varphi_{x}\left(K_{1}\right)=K .
\end{array}
$$


Let $\left(V_{i}, \pi_{i}\right)$ be representations of $U_{q}$ with the highest weights $\lambda_{i}$. Then $\left(V_{i}(x), \hat{\pi}_{i}\right)=\left(V_{i}, \pi_{i} \circ \varphi_{x}\right)$ gives a representation of $\hat{U}_{q}$ for each $x \in \mathbf{C}$. The operator

$$
R_{V_{i} V_{j}}(x): V_{i}(x) \otimes V_{j}(1) \rightarrow V_{i}(x) \otimes V_{j}(1)
$$

such that

$$
\Delta^{\prime}(a) R_{V_{i} V_{j}}(x)=R_{V_{\imath} V_{j}}(x) \Delta(a), \quad a \in \hat{U}_{q}
$$

gives a trigonometric $R$-matrix. Let $v_{i}$ be the highest weight vector in $V_{i}$. We fix a choice of normalization such that

$$
R_{V_{i} V_{j}}(x) v_{i} \otimes v_{j}=v_{i} \otimes v_{j} .
$$

Then $R_{V_{i} V_{j}}(x)$ acts as

$$
\begin{aligned}
& R_{V_{\imath} V_{j}}(x) X^{-} v_{i} \otimes v_{j}=\frac{x q^{m_{j}}-q^{m_{i}}}{x-q^{m_{i}+m_{j}}} X^{-} v_{i} \otimes v_{j}+\frac{1-q^{2 m_{j}}}{x-q^{m_{i}+m_{j}}} v_{i} \otimes X^{-} v_{j} \\
& R_{V_{i} V_{j}}(x) v_{i} \otimes X^{-} v_{j}=\frac{x\left(1-q^{2 m_{i}}\right)}{x-q^{m_{i}+m_{j}}} X^{-} v_{i} \otimes v_{j}+\frac{x q^{m_{i}}-q^{m_{j}}}{x-q^{m_{i}+m_{j}}} v_{i} \otimes X^{-} v_{j} .
\end{aligned}
$$

Here $m_{i}=\left(\lambda_{i}, \alpha\right)$ for the simple root $\alpha$.

Let $\lambda_{1}, \ldots, \lambda_{n}, \lambda$ be a set of weights. Let $V_{i}$ be the irreducible representation of $U_{q}$ with the highest weight $\lambda_{i}$ and the highest weight vector $v_{i}$. Let $v$ be a complex parameter and put $p^{v}=q$. We set $\rho=\alpha / 2$, the half sum of the positive roots. For a weight $\mu$, we denote by $\left(q^{\mu}\right)_{k}$ the action of $q^{\mu}$ on the $k^{\text {th }}$ component of the tensor product $V_{1} \otimes \ldots \otimes V_{n}$. For instance,

$$
q^{\mu}\left(v_{k}\right)=q^{\left(\mu, \lambda_{k}\right)} v_{k}, \quad q^{\mu}\left(X^{-} v_{k}\right)=q^{\left(\mu, \lambda_{k}-\alpha\right)} X^{-} v_{k} .
$$

The quantum Knizhnik-Zamolodchikov equation introduced by Frenkel and Reshetikhin [FR] is written as the following system of $q$-difference equations:

$$
\begin{aligned}
T_{k} \mathscr{F}= & R_{V_{k} V_{k-1}}\left(p x_{k} / x_{k-1}\right) \ldots R_{V_{k} V_{1}}\left(p x_{k} / x_{1}\right)\left(q^{\lambda+2 \rho}\right)_{k} \\
& \times q^{-\left(\lambda, \lambda_{k}\right)} R_{V_{k+1} V_{k}}\left(x_{k+1} / x_{k}\right)^{-1} \ldots R_{V_{n} V_{k}}\left(x_{n} / x_{k}\right)^{-1} \mathscr{F}, \\
k= & 1, \ldots, n,
\end{aligned}
$$

where $\mathscr{F}=\mathscr{F}\left(x_{1}, \ldots, x_{n}\right)$ is a function valued in $V_{1} \otimes \ldots \otimes V_{n}$.

Let us compare Eqs. (2.12) and (3.9). Take the weights $\lambda_{0}, \lambda_{\infty}$ such that

$$
\lambda_{0}+\cdots+\lambda_{n}-\lambda_{\infty}=\alpha, \quad \lambda_{0}+\lambda_{\infty}=\lambda,
$$

and put the parameters as:

$$
\beta=-2\left(\lambda_{\infty}+\alpha, \alpha\right) v, \quad \beta_{i}=2\left(\lambda_{i}, \alpha\right) v
$$

We set

$$
\varphi_{i}\left(x_{1}, \ldots, x_{n}\right)=p^{\left(\beta_{i+1}+\cdots+\beta_{n}\right) / 2} x_{1}^{\beta_{1}} \ldots x_{n}^{\beta_{n}} F_{i}\left(p^{\beta_{1} / 2} x_{1}, \ldots, p^{\beta_{n} / 2} x_{n}\right),
$$

for each $i=1, \ldots, n$, and define the $V_{1} \otimes \ldots \otimes V_{n}$-valued function $\mathscr{F}$ by

$$
\mathscr{F}=\sum_{i=1}^{n} \varphi_{i}\left(x_{1}, \ldots, x_{n}\right) v_{1} \otimes \ldots \otimes X^{-} v_{i} \otimes \ldots \otimes v_{n} .
$$

Then, by rewriting Eq. (2.12) in terms of $\mathscr{F}$, we have 
Theorem 3. The system (2.12) is equivalent to the restriction of the system (3.9) to the weight subspace with the weight $\lambda_{1}+\cdots+\lambda_{n}-\alpha$, and the function $\mathscr{F}$ defined by (3.13) is a solution of (3.9).

Remark. When $q$ goes to $1, \mathscr{F}$ defined by (3.13) goes to a special case of the integral solutions to the Knizhnik-Zamolodchikov equation obtained by Cherednik [ $\mathrm{Ch}$ ] in the trigonometric form.

We shall give another description of the equation. Let $\lambda_{0}, \ldots, \lambda_{n}, \lambda_{\infty}$ be a set of weights such that

$$
\lambda_{0}+\cdots+\lambda_{n}-\lambda_{\infty}=\alpha .
$$

Let $V_{i}$ be the irreducible representation of $U_{q}$ with the highest weight $\lambda_{i}$ and the highest weight vector $v_{i}$. The quantum Knizhnik-Zamolodchikov equation for a $\operatorname{Hom}_{U_{q}}\left(V_{\infty}, V_{0} \otimes \ldots \otimes V_{n}\right)$-valued function $\mathscr{F}$ is written as:

$$
\begin{aligned}
T_{k} \mathscr{F}= & R_{V_{k} V_{k-1}}\left(p x_{k} / x_{k-1}\right) \ldots R_{V_{k} V_{1}}\left(p x_{k} / x_{1}\right) R_{V_{k} V_{0}}(0)\left(q^{2 \rho}\right)_{k} \\
& \times R_{V_{\infty}^{*} V_{k}}(0)^{-1} R_{V_{k+1} V_{k}}\left(x_{k+1} / x_{k}\right)^{-1} \ldots R_{V_{n} V_{k}}\left(x_{n} / x_{k}\right)^{-1} \mathscr{F} .
\end{aligned}
$$

Here we understand $\mathscr{F}$ as an element of $V_{0} \otimes \ldots \otimes V_{n} \otimes V_{\infty}^{*}$. Next we consider the set $\mathscr{H}_{\lambda_{\infty}}\left(V_{0} \otimes \ldots \otimes V_{n}\right)$ of highest weight vectors in $V_{0} \otimes \ldots \otimes V_{n}$ with the weight $\lambda_{\infty}$. We have an injection

$$
\operatorname{Hom}_{U_{q}}\left(V_{\infty}, V_{0} \otimes \ldots \otimes V_{n}\right) \rightarrow \mathscr{H}_{\lambda_{\infty}}\left(V_{0} \otimes \ldots \otimes V_{n}\right)
$$

by evaluating the highest weight vector $v_{\infty}$. Then Eq. (3.15) is regarded as a restriction of the following system:

$$
\begin{aligned}
T_{k} \mathscr{F}= & R_{V_{k} V_{k-1}}\left(p x_{k} / x_{k-1}\right) \ldots R_{V_{k} V_{1}}\left(p x_{k} / x_{1}\right) R_{V_{k} V_{0}}(0)\left(q^{\lambda_{\infty}+2 \rho}\right)_{k} q^{-\left(\lambda_{\infty}, \lambda_{k}\right)} \\
& \times R_{V_{k+1} V_{k}}\left(x_{k+1} / x_{k}\right)^{-1} \ldots R_{V_{n} V_{k}}\left(x_{n} / x_{k}\right)^{-1} \mathscr{F}
\end{aligned}
$$

where $\mathscr{F}$ is a $\mathscr{H}_{\lambda_{\infty}}\left(V_{0} \otimes \ldots \otimes V_{n}\right)$-valued function.

Remarks. (1) If all $V_{i}$ are the Verma modules or are the finite dimensional modules, then the linear map (3.16) is surjective, and the system (3.15) is the same as (3.17).

(2) If $q^{2\left(\lambda_{0}, \alpha\right)} \neq 1$, then the system (3.17) is same as the restriction of the system (3.9) to the weight subspace with the weight $\lambda_{1}+\cdots+\lambda_{n}-\alpha$, hence is equivalent to the system (2.12).

We define the $V_{0} \otimes \ldots \otimes V_{n}$-valued function $\mathscr{F}$ by

$$
\mathscr{F}=\sum_{i=0}^{n} \varphi_{i}\left(x_{1}, \ldots, x_{n}\right) v_{0} \otimes \ldots \otimes X^{-} v_{i} \otimes \ldots \otimes v_{n},
$$

where $\varphi_{i}$ is defined by (3.12) for each $i=0, \ldots, n$. Then, by interpreting the identity (2.17), we have

$$
X^{+} \mathscr{F}=0
$$

Therefore $\mathscr{F}$ is one of the highest weight vectors in $V_{0} \otimes \ldots \otimes V_{n}$ with the weight $\lambda_{\infty}$. Thus we finally obtain:

Theorem 4. The $\mathscr{H}_{\lambda_{\infty}}\left(V_{0} \otimes \ldots \otimes V_{n}\right)$-valued function $\mathscr{F}$ defined by (3.18) is a solution of the quantum Knizhnik-Zamolodchikov equation (3.17). 
Notes. (1) In the situation of [FR], $V_{0}$ and $V_{\infty}$ are integrable $\hat{U}_{q}$-modules and $V_{1}, \ldots, V_{n}$ are finite dimensional $\hat{U}_{q}$-modules, and $v$ corresponds to $-\frac{1}{2(k+g)}$, where $k$ is the fixed level and $g$ is the dual coxeter number. Moreover the quantum Knizhnik-Zamolodchikov equation for the correlation function is written in terms of the image of the universal $R$-matrix, which differs from our $R_{V_{i} V_{j}}$ by a certain scalar factor.

(2) For $n=2$, our expressions of solutions to (3.9) coincide with those given in [FR, Sect. 7].

\section{Proof of Propositions}

We write $\phi_{1}(t) \sim \phi_{2}(t)$ if

$$
\int_{0}^{s \infty} \phi_{1}(t) d_{p} t=\int_{0}^{s \infty} \phi_{2}(t) d_{p} t
$$

holds for any $s \in \mathbf{C}^{*}$. For example, we have

$$
\Phi_{i}(t) \sim p \Phi_{i}(p t) .
$$

Proof of Proposition 1. The following is obvious from the definition:

$$
T_{k} F_{i}=\int_{0}^{s \infty} T_{k} \Phi_{i}(t) d_{p} t
$$

Therefore the $q$-difference system (2.12) is equivalent to

$$
T_{k} \Phi_{j}(t) \sim \sum_{i=1}^{n} a_{i j}^{k} \Phi_{i}(t) .
$$

Now, because of (4.2), the following lemma is enough to prove the proposition.

\section{Lemma 5.}

(a) For $j<k$, we have

$$
p T_{k} \Phi_{j}(p t)=p \sum_{i=1}^{j} a_{i j}^{k} \Phi_{i}(p t)+\sum_{i=k}^{n} a_{i j}^{k} \Phi_{i}(t) .
$$

(b) For $j=k$, we have

$$
p T_{k} \Phi_{j}(p t)=p \sum_{i=1}^{j-1} a_{i j}^{k} \Phi_{i}(p t)+\sum_{i=j}^{n} a_{i j}^{k} \Phi_{i}(t) .
$$

(c) For $k<j$, we have

$$
T_{k} \Phi_{j}(t)=\sum_{i=k}^{j} a_{i j}^{k} \Phi_{i}(t) .
$$

Proof. Since all the cases are treated in a similar way, we will exhibit detailed calculations only for the most difficult case (b). We put $a_{i j}=a_{i j}^{k}$ for simplicity. 
Multiplied by appropriate factors, (b) is equivalent to

$$
\begin{aligned}
& p^{\beta} x_{j} \prod_{l=1}^{j-1}\left(p^{\beta_{l}} p t-x_{l}\right) \prod_{l=j+1}^{n}\left(p^{\beta_{l}} t-x_{l}\right) \\
& =p^{\beta} \sum_{i=1}^{j-1} a_{i j} x_{i} \prod_{l=1}^{i-1}\left(p^{\beta_{l}} p t-x_{l}\right) \prod_{l=i+1}^{j-1}\left(p t-x_{l}\right) \prod_{l=j}^{n}\left(p^{\beta_{l}} t-x_{l}\right) \\
& \quad+\sum_{i=j}^{n} a_{i j} x_{i} \prod_{l=1}^{j-1}\left(p t-x_{l}\right) \prod_{l=j}^{i-1}\left(p^{\beta_{l}} t-x_{l}\right) \prod_{l=i+1}^{n}\left(t-x_{l}\right) .
\end{aligned}
$$

Since both sides are polynomials of degree $n-1$ with respect to $t$, it suffices to check the equality at $n$ different values of $t$. Putting $t=x_{m} / p$ for $m \leqq j-1$ in (4.5), we have

$$
p x_{j} \prod_{l=m}^{j-1}\left(p^{\beta_{l}} x_{m}-x_{l}\right)-\sum_{i=m}^{j-1} a_{i j} x_{i}\left(p^{\beta_{j}} x_{m}-p x_{j}\right) \prod_{l=m}^{i-1}\left(p^{\beta_{l}} x_{m}-x_{l}\right) \prod_{l=i+1}^{j-1}\left(x_{m}-x_{l}\right)=0 .
$$

We put $t=x_{j} / p^{\beta_{j}}$, then we have

$$
\begin{gathered}
p^{\beta} \prod_{l=1}^{j-1}\left(p^{\beta_{l}} p x_{j}-p^{\beta_{j}} x_{l}\right) \prod_{l=j+1}^{n}\left(p^{\beta_{l}} x_{j}-p^{\beta_{j}} x_{l}\right) \\
=a_{j j} \prod_{l=1}^{j-1}\left(p x_{j}-p^{\beta_{j}} x_{l}\right) \prod_{l=j+1}^{n}\left(x_{j}-p^{\beta_{j}} x_{l}\right) .
\end{gathered}
$$

We finally put $t=x_{m} / p^{\beta_{m}}$ for $j+1 \leqq m$, then we have

$$
\sum_{i=j}^{m} a_{i j} x_{i} \prod_{l=j}^{i-1}\left(p^{\beta_{l}} x_{m}-p^{\beta_{m}} x_{l}\right) \prod_{l=i+1}^{n}\left(x_{m}-p^{\beta_{m}} x_{l}\right)=0 .
$$

Now let us consider the explicit values of $a_{i j}$ defined by (2.8)-(2.10). Substitute them in the left of (4.6) inductively as $i=j-1, j-2, \ldots, N$. Then we have

$$
\begin{gathered}
p x_{j} \prod_{l=N}^{j-1} \frac{p^{\beta_{l}} p x_{j}-p^{\beta_{j}} x_{l}}{p x_{j}-p^{\beta_{j}} x_{l}} \prod_{l=m}^{N-1}\left(p^{\beta_{l}} x_{m}-x_{l}\right) \prod_{l=N}^{j-1}\left(x_{m}-x_{l}\right) \\
-\sum_{i=m}^{N} a_{i j} x_{i}\left(p^{\beta_{j}} x_{m}-p x_{j}\right) \prod_{l=m}^{i-1}\left(p^{\beta_{l}} x_{m}-x_{l}\right) \prod_{l=i+1}^{j-1}\left(x_{m}-x_{l}\right) .
\end{gathered}
$$

When $N=m$, this is zero and (4.6) is verified. Equation (4.7) follows easily from (2.10). To verify (4.8), it suffices to substitute the values of $a_{i j}, i=j, j+1, \ldots, N$ inductively. Hence (4.5) is shown and the proof of (b) is completed.

Q.E.D.

Proof of Proposition 2. By the relation (4.2), it suffices to show the following lemma.

Lemma 6. We have the following relation:

$$
p^{\beta_{1}+\cdots+\beta_{n}} \Phi_{0}(t)-p^{-\beta+1} \Phi_{0}(p t)=\sum_{i=1}^{n} p^{\beta_{i+1}+\cdots+\beta_{n}}\left(p^{\beta_{i}}-1\right) \Phi_{i}(t) .
$$


Proof. Multiplied by an appropriate factor, (4.9) is equivalent to

$$
\begin{aligned}
p^{\beta_{1}+\cdots+\beta_{n}} & \prod_{j=1}^{n}\left(1-t / x_{j}\right)-\prod_{j=1}^{n}\left(1-p^{\beta_{j}} t / x_{j}\right) \\
= & \sum_{i=1}^{n} p^{\beta_{i+1}+\cdots+\beta_{n}}\left(p^{\beta_{i}}-1\right) \prod_{j=1}^{i-1}\left(1-p^{\beta_{j}} t / x_{j}\right) \prod_{j=i+1}^{n}\left(1-t / x_{j}\right) .
\end{aligned}
$$

The right becomes

$$
\begin{aligned}
& \sum_{i=1}^{n} p^{\beta_{i}+\cdots+\beta_{n}} \prod_{j=1}^{i-1}\left(1-p^{\beta_{j}} t / x_{j}\right) \prod_{j=i+1}^{n}\left(1-t / x_{j}\right) \\
& \quad \times \sum_{i=1}^{n} p^{\beta_{i+1}+\cdots+\beta_{n}} \prod_{j=1}^{i-1}\left(1-p^{\beta_{j}} t / x_{j}\right) \prod_{j=i+1}^{n}\left(1-t / x_{j}\right) \\
& =\sum_{i=1}^{n} p^{\beta_{i}+\cdots+\beta_{n}} \prod_{j=1}^{i-1}\left(1-p^{\beta_{j}} t / x_{j}\right) \prod_{j=i}^{n}\left(1-t / x_{j}\right) \\
& \quad \times \sum_{i=1}^{n} p^{\beta_{i+1}+\cdots+\beta_{n}} \prod_{j=1}^{i}\left(1-p^{\beta_{j}} t / x_{j}\right) \prod_{j=i+1}^{n}\left(1-t / x_{j}\right),
\end{aligned}
$$

which yields the left of (4.10).

Q.E.D.

\section{Discussions}

In this paper, we have constructed a Jackson integral representation of solutions to the quantum Knizhnik-Zamolodchikov equation in the simplest case for $U_{q}\left(\widehat{\mathfrak{s l}}_{2}\right)$. Let us briefly review the results of [AKM] and [FR], and discuss the relation of our result and the connection problem of $q$-difference equations.

Let $F_{i}^{\prime}=F_{i}^{\prime}\left(x_{1}, \ldots, x_{n}\right)$ be the function defined by

$$
F_{i}^{\prime}=\int_{0}^{s \infty} \frac{t^{\beta-1}}{1-t / x_{i}} \frac{\prod_{j=1}^{n}\left(t / x_{j}\right)_{\infty}}{\prod_{j=1}^{n}\left(p^{\beta_{j}} t / x_{j}\right)_{\infty}} d_{q} t
$$

Consider the system satisfied by $F_{i}^{\prime}$ :

$$
\left(T_{k} F_{1}^{\prime}, \ldots, T_{k} F_{n}^{\prime}\right)=\left(F_{1}^{\prime}, \ldots, F_{n}^{\prime}\right) A_{k}^{\prime} .
$$

The asymptotic behavior in

$$
\left\{\left(x_{1}, \ldots, x_{n}\right) ;\left|x_{\sigma(1)}\right| \gg \cdots \gg\left|x_{\sigma(n)}\right| \gg 1\right\}
$$

characterizes the fundamental solution $\Xi_{\sigma}=\Xi_{\sigma}\left(x_{1}, \ldots, x_{n}\right)$ for a permutation $\sigma \in \Xi_{n}$. Let $e$ be the identity in $\Im_{n}$. In the sense of [Mi], the elementary connection matrix $P_{i}$ is defined by $\Xi_{\sigma_{i}}=P_{i} \Xi_{e}$ for a transposition $\sigma_{i}=(i, i+1) \in \Xi_{n}$. Then it is shown in [AKM], for $\beta_{1}=\cdots=\beta_{n}$, that $P_{i}$ depends only on the ratio $x_{i} / x_{i+1}$ and satisfies the Yang-Baxter equation:

$$
P_{i}(u) P_{i+1}(u v) P_{i}(v)=P_{i+1}(v) P_{i}(u v) P_{i+1}(u) .
$$


This is equivalent to the Boltzmann weights of the eight vertex SOS model, i.e., the $\mathrm{ABF}$-solution of the star-triangle relation (cf. [ABF, JMO]).

On the other hand, Frenkel and Reshetikhin [FR] studied a $q$-deformed chiral vertex operator along the line of $[\mathrm{TK}]$, for a quantum affine algebra $U_{q}(\hat{\mathrm{g}})$. They showed that the correlation function satisfies the quantum KnizhnikZamolodchikov equation, which is written in terms of the universal $R$-matrix, and considered the connection matrix as a $q$-analogue of the braiding matrix in conformal field theory. In some situations, they proved that the connection matrix of the quantum Knizhnik-Zamolodchikov equation for a simple transposition depends only on the ratio of two arguments and it satisfies the quantum Yang-Baxter equation. The most remarkable point of their theory is the factorization property, from which it is possible to determine the connection matrix by computing it for $n=2$, namely by considering the 4-point function as in the discussion of [TK]. Using this argument and considering Jackson integral solutions for $n=2$, they calculated the connection matrix in the simplest case for $U_{q}\left(\widehat{\mathfrak{s l}}_{2}\right)$ which includes the ABF-solution [FR, Sect. 7]. Therefore the connection matrix of the quantum Knizhnik-Zamolodchikov equation for a special case coincides with that of [AKM].

Now our Eq. (2.12) for the function $F_{i}$ defined by (2.5) is obviously equivalent to Eq. (5.1). In fact, $F_{i}$ and $F_{i}^{\prime}$ are related to each other by a triangular matrix:

$$
F_{i}=\sum_{j=1}^{i} b_{i j} F_{j}^{\prime}
$$

The explicit form is given by

$$
b_{i j}=\prod_{k=1}^{i} b_{i j}^{k}, \quad b_{i j}^{k}= \begin{cases}\frac{p^{\beta_{j}} x_{j}-x_{k}}{x_{j}-x_{k}} & (\text { if } k<i) \\ \frac{\left(p^{\beta_{i}}-1\right) x_{i}}{x_{j}-x_{i}} & (\text { if } k=i) .\end{cases}
$$

Since Theorem 3 says that Eq. (2.12) is equivalent to the quantum KnizhnikZamolodchikov equation (3.9), we have seen the coincidence above explicitly at the level of the $q$-difference equation before going to the connection matrix. Finally, combined with the discussions in [FR], the results in the present paper enable us to observe the surprising phenomenon revealed by [AKM], that a very rich structure is contained in such a simple expression:

$$
\int_{0}^{s \infty} t^{\beta-1} \prod_{1 \leqq j \leqq n} \frac{\left(t / x_{j}\right)_{\infty}}{\left(p^{\beta_{j}} t / x_{j}\right)_{\infty}} d_{q} t,
$$

from the viewpoint of the representation theory of quantum enveloping algebra $U_{q}\left(\widehat{\mathfrak{s l}}_{2}\right)$. 


\section{References}

[A1] Aomoto, K.: On the structure of integrals of power product of linear functions. Sci. Papers. Coll. Gen. Ed. Univ. Tokyo 27, 49 (1977)

[A2] Aomoto, K.: Gauss-Manin connection of integrals of difference products. J. Math. Soc. Jpn. 39, 191 (1987)

[A3] Aomoto, K.: Finiteness of a cohomology associated with certain Jackson integrals. Tohoku Math. J. 43, 75 (1991)

[ABF] Andrews, G.E., Baxter, R.J., Forrester, P.J.: Eight-vertex SOS model and generalized Rogers-Ramanujan-type identities. J. Stat. Phys. 35, 193 (1984)

[AKM] Aomoto, K., Kato, Y., Mimachi, K.: A solution of Yang-Baxter equation as connection coefficients of a holonomic $q$-difference system. Preprint

[ATY] Awata, H., Tsuchiya, A., Yamada, Y.: Integral formulas for the WZNW correlation functions. Preprint KEK-TH-286

[Ch] Cherednik, I.V.: Integral solutions to the trigonometric Knizhnik-Zamolodchikov equations and Kac-Moody algebras. Publ. RIMS 27, 727 (1991)

[DJMM] Date, E., Jimbo, M., Matsuo, A., Miwa, T.: Hypergeometric type integrals and the sl(2, C) Knizhnik-Zamolodchikov equations. In: Yang-Baxter Equations, Conformal Invariance and Integrability in Statistical Mechanics and Field Theory. Singapore: World Scientifc

[D] Drinfeld, V.G.: Quasi-Hopf algebras. Algebras Anal. 1, 30 (1990)

[FR] Frenkel, I.B., Reshetikhin, N.Yu.: Quantum affine algebras and holonomic difference equations. Preprint

[JMO] Jimbo, M., Miwa, T., Okado, M.: Solvable lattice models related to the vector representation of classical simple Lie algebra. Commun. Math. Phys. 116, 507 (1988)

[KZ] Knizhnik, V.G., Zamolodchikov, A.B.: Current algebra and Wess-Zumino models in two dimensions. Nucl. Phys. B247, 83 (1984)

[Ko] Kohno, T.: Monodromy representations of braid groups and Yang-Baxter equations. Ann. Inst. Fourier Grenouble 37, 139 (1987)

[Ku] Kuroki, G.: Fock space representations of affine Lie algebras and integral representations in the Wess-Zumino-Witten model. Commun. Math. Phys. 142, 511 (1991)

[Ma] Matsuo, A.: An application of Aomoto-Gelfand hypergeometric functions to $S U(n)$ Knizhnik-Zamolodchikov equation. Commun. Math. Phys. 134, 65 (1990)

[Mi] Mimachi, K.: Connection problem in holonomic $q$-difference system associated with a Jackson integral of Jordan-Pochhammer type. Nagoya Math. J. 116, 149 (1989)

[SV] Schechtman, V.V., Varchenko, A.N.: Arrangement of hyperplanes and Lie algebra homology. Invent. Math. 106, 139 (1991)

[TK] Tsuchiya, A., Kanie, Y.: Vertex operators in conformal field theory on $\mathbf{P}^{1}$ and monodromy representations of braid groups. Adv. Stud. Pure. Math 16, 297 (1988) 
